\title{
Theoretical perspective for the future experiments on parton densities
}

\author{
S. Kumano ${ }^{a, b}$ \\ ${ }^{a}$ KEK Theory Center, Institute of Particle and Nuclear Studies, KEK, \\ and Department of Particle and Nuclear Physics, Graduate University for Advanced Studies \\ (SOKENDAI), Ooho 1-1, Tsukuba, Ibaraki, 305-0801, Japan \\ ${ }^{b}$ J-PARC Branch, KEK Theory Center, Institute of Particle and Nuclear Studies, KEK, \\ and Theory Group, Particle and Nuclear Physics Division, J-PARC Center, \\ 203-1, Shirakata, Tokai, Ibaraki, 319-1106, Japan
}

E-mail: Shunzo.kumanodkek.jp

\begin{abstract}
I explain the current status of parton-distribution-function (PDF) studies and future experimental prospects on their determinations. First, unpolarized PDFs of the nucleon are introduced as a field of precision QCD physics including higher-order $\alpha_{s}$ corrections. Second, nuclear PDFs and polarized nucleonic PDFs are discussed. Third, the determination of fragmentation functions is explained. Forth, the three-dimensional (3D) structure functions are discussed in connection with the origin of the nucleon spin and gravitational form factors of hadrons. By the 3D structure functions, gravitational sources and the origin of nucleon mass could be clarified in the microscopic level of quarks and gluons. Furthermore, 3D structure studies of hadrons could be used for clarifying internal structure of exotic hadron candidates, nuclear composition of ultra-high-energy cosmic rays, and color-entanglement phenomena. The PDF field will be developed further along with progress in other fields of science.
\end{abstract}

XXVI International Workshop on Deep-Inelastic Scattering and Related Subjects (DIS2018) 16-20 April 2018

Kobe, Japan 


\section{Introduction}

Hadron physics is the field of science to study material creation in the universe and properties of quark-hadron many-body systems with ultimate densities in nature. We know that the basic theory for strong interactions is Quantum Chromodynamics (QCD), which describes quark and gluon interactions. Studies of the parton distribution functions (PDFs) of hadrons are a part of our efforts for universal understanding of the quark-hadron many-body systems from low to high densities, from low to high temperatures, and from low to high energies.

The PDFs indicate parton momentum distributions inside a hadron, and they play an important role in calculating high-energy hadron cross sections precisely as shown typically in Fig. W. Without their precise determination, it is impossible to find any new physics beyond the current standard model and any new phenomenon in high-energy lepton-hadron and hadron-hadron reactions, for example, at LHC (Large Hadron Collider). Thank to significant theoretical and experimental efforts on high-energy hadron reactions including higher-order $\alpha_{s}$ corrections, QCD became a field of precision physics, roughly speaking, within a several percent level, although the precision depends on kinematical regions as one will find later as uncertainty bands of the PDFs.

Recent PDF studies are not only on the traditional unpolarized nucleonic PDFs but also on nuclear PDFs, polarized PDFs, fragmentation functions, and 3-dimensional structure functions. As the nucleonic PDFs become more and more accurate, nuclear corrections cannot be ignored anymore if nuclear data are partially included in the "nucleonic" PDF analysis. The corrections are of the order of 10-30\% for medium and large nuclei. The nuclear PDFs are valuable in finding new phenomena in high-energy heavy-ion collisions and also in high-precision neutrino oscillation experiments. The polarized PDFs are essential for understanding the origin of nucleon spin, which is one of fundamental physics quantities. We found that orbital-angular-momentum contributions of partons could become a significant part of the nucleon spin, and they should be investigated by the three-dimensional structure functions such as the generalized parton distributions (GPDs). In addition to inclusive lepton and hadron reactions, semi-inclusive processes, e.g. pion production, become usual tools in investigating the internal structure of hadrons, such as flavor dependence of the PDFs and transverse-momentum-dependent parton distribution (TMDs), and quark-gluon plasma properties. For descriptions of high-energy hadron productions, accurate fragmentation functions are essential.

In this article, the current status and prospects are discussed on these topics by considering

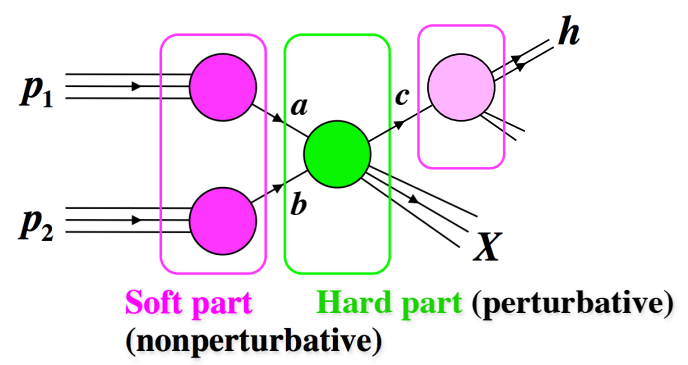

Figure 1: High-energy hadron reactions.

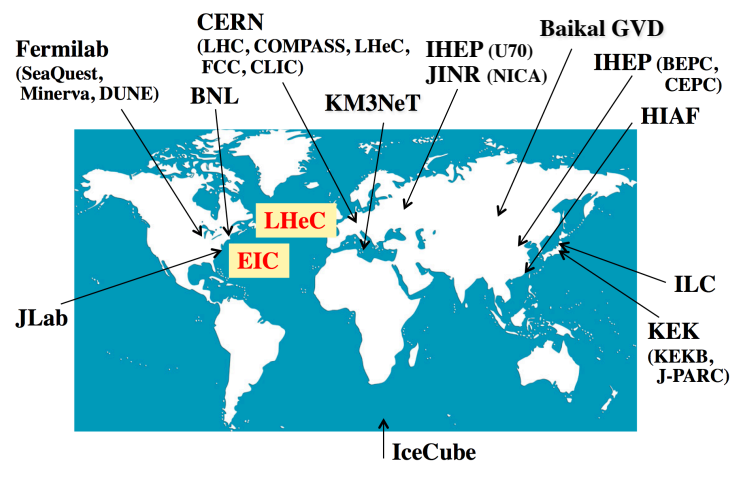

Figure 2: Current and future facilities. 
future experimental projects. For the DIS (deep inelastic scattering) community of this conference, the EIC (Electron-Ion Collider) and LHeC (Large Hadron Electron Collider) are two major important projects in the middle of 2020's. Here in Japan, there are projects of KEKB, JPARC (Japan Proton Accelerator Research Complex), and ILC (International Linear Collider) for structure-function and fragmentation-function studies as explained in this article. In addition, there are ongoing and future world-wide projects at Jefferson Lab $12 \mathrm{GeV}$, CERN (COMPASS, LHC, FCC, CLIC), Fermilab (Sea-Quest, Minerva, DUNE), BNL (RHIC), Chinese IHEP (BEPC, CEPC), HIAF, Russian IHEP (U70), JINR (NICA), KM3NeT, and Baikal GVD as shown in Fig. $\square$. In Sec.】, the unpolarized PDFs of the nucleon are discussed, and their nuclear corrections are explained in Sec. B]. The polarized PDFs, fragmentation functions, and 3D structure functions are discussed in Secs. 团, 且, and 耳, respectively. These discussions are summarized in Sec.D.

\section{Unpolarized PDFs of the nucleon}

The unpolarized PDFs of the nucleon have been investigated for a long time, and this topic is considered as a precision QCD field. As the higher-order $\alpha_{s}$ corrections are now included in the NNLO (next-to-next to leading order) level as a standard [四], and the $\mathrm{N}^{3} \mathrm{LO}$ evolution and coefficient functions are within reach of theoretical studies [ [D]. The PDFs are determined by a global $\chi^{2}$ analysis of high-energy hadron reaction data. They are expressed by a number of parameters at a fixed $Q^{2}$, and they are evolved to experimental $Q^{2}$ points to calculate $\chi^{2}$ by the DGLAP (Dokshitzer-Gribov-Lipatov-Altarelli-Parisi) evolution equations including higher-order corrections. Recently, another analysis of using a neutral-network method has been in progress, and it could be a reliable error estimate of the PDFs because it

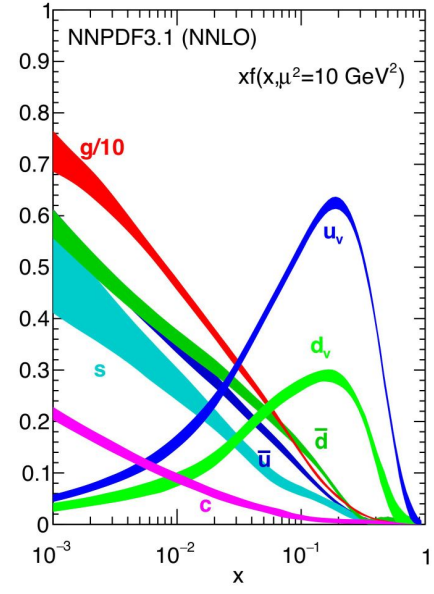

Figure 3: Recent PDFs [四]. does not rely on a specific functional form.

Recent typical PDFs are shown in Fig. [3] with error bands. Now, the distributions are well determined from small Bjorken- $x\left(\sim 10^{-3}\right)$ to large $x$ in the NNLO. The strange-quark distribution has been determined by the neutrino-induced opposite-sign dimuon measurements. Roughly, it is about $40 \%$ of the light antiquark distributions, $s \sim 0.4(\bar{u}+\bar{d}) / 2$ at small $Q^{2}$. The CMS measurements on $W$ and charm production agree with this strange-quark distribution. However, the ATLAS collaboration suggested that $s$ is similar to the light antiquark distributions. There were discussions in the parallel sessions of this conference including analysis method. It may take a few yeas to resolve this issue.

As the PDFs become more and more accurate, we hope that the longstanding issue of $\mathrm{NuTeV}$ anomaly could be solved. The $\mathrm{NuTeV}$ anomaly indicates the weak-mixing-angle difference for the $\mathrm{NuTeV}$ neutrino DIS measurement from other data average. It is obtained in the $\mathrm{NuTeV}$ analysis by using the Paschos-Wolfenstein relation for neutral- and charged-current neutrino cross sections [B]. However, we need to take into account various small correction factors to this relation: $R_{A}^{-}=$ $\left(\sigma_{N C}^{V A}-\sigma_{N C}^{\bar{v} A}\right) /\left(\sigma_{C C}^{V A}-\sigma_{C C}^{\bar{\nu} A}\right)=1 / 2-\sin ^{2} \theta_{W}+O\left(\varepsilon_{n}\right)+O\left(\varepsilon_{i}, \varepsilon_{v}, \varepsilon_{s_{v}}, \varepsilon_{c_{v}}\right)+\cdots$. Here, $\varepsilon_{n}$ is the neutronexcess correction in the iron nucleus and it was taken into account in the $\mathrm{NuTeV}$ analysis. However, 
the other corrections could be the source of the $\mathrm{NuTeV}$ anomaly. The $\varepsilon_{i}$ is the isospin-violation effects on the PDFs, $\varepsilon_{v}$ is the nuclear modification difference between $u_{v}$ and $d_{v}$ in the iron, $\varepsilon_{s_{v}}\left(\varepsilon_{c_{v}}\right)$ is the valence strange (charm) quark $\left(q_{v} \equiv q-\bar{q}\right)$ effect. Of course, there is no net strangeness and charm in the nucleon and ordinary nuclei, so that $\int d x s_{v}(x)=\int d x c_{v}(x)=0$ should be satisfied, but it does not mean no $x$ dependence: $s_{v}(x) \neq 0$ and $c_{v}(x) \neq 0$. All of these corrections are difficult to be determined at this stage; however, studies of accurate PDFs from both theoretical and experimental sides could lead to a finding on the source for the $\mathrm{NuTeV}$ anomaly.

There is recent progress on the PDFs in lattice QCD [䧃]. The PDFs are defined by matrix elements of two-field correlators with gauge links between the fields to satisfy the color gauge invariance. Instead of lightcone-separated field correlators, we may define quasi-PDFs with an equal-time separation, so that it becomes possible to calculate the quasi-PDFs by lattice QCD. Both PDFs agree in the infinite momentum limit $p_{z} \rightarrow \infty$. This is a numerically challenging project for obtaining the PDFs at large $p_{z}$ in lattice QCD; however, it could be a promising future direction of theoretical PDFs from QCD. In particular, if there is no sufficient experimental information on some quantities, the lattice QCD studies will provide us a guideline.

As for the future prospect of the unpolarized PDFs of the nucleon, we expect that the PDFs will be improved steadily with measurements of LHC and future experimental data of EIC and LHeC. Another interesting prospect is to study a real global analysis with not only the unpolarized nucleonic data but also together with nuclear and polarized ones. On the other hand, it is interesting to test the current PDFs in ultra-high energy reactions which cannot be attained by man-made accelerators. There exist ultra-high energy cosmic rays which arrive on the earth. By extending our current knowledge of high-energy hadron reactions, air-shower codes have been developed. The leading shower development is dominated by forward physics, namely described mainly by the Regge and Pomeron theories. It is an interesting topic; however, for a direct connection with the PDFs, we could test them by cross sections of ultra-high energy neutrinos. The first IceCube neutrino cross sections were recently reported up to the $10^{15} \mathrm{eV}$ range [[]]. Although the IceCube cross sections are slightly larger than the standard-model estimates, they are consistent within experimental uncertainties.

\section{Nuclear PDFs}

In 1970's, there was a prejudice that nuclear effects, which are small energy-momentum scale phenomena of the order of 10-100 MeV, do not change DIS structure functions measured in the range of 10-100 GeV. Although nucleon Fermi-motion effects were noticed at the early stage, the EMC (European Muon Collaboration) discovery on nuclear modification of $F_{2}$ was rather surprising. It used to be considered as the first finding on an explicit quark signature in nuclear physics. However, it is generally very difficult to pin down such an effect even though nuclei are dense systems of nucleons with the average nucleon separation $2.2 \mathrm{fm}$, which is almost equal to the nucleon diameter $1.8 \mathrm{fm}$. It was found later that usual nuclear mechanisms, in terms of nuclear binding and Fermi motion, could describe the major part of the nuclear modifications of $F_{2}$ at $x>0.2$. Therefore, although the EMC finding may contain important discoveries, it is not easy to draw a solid conclusion on a new hadron-physics mechanism. 
In any case, the nuclear modifications of the PDFs should be understood accurately for practical purposes, for example, in order to investigate properties of quark-gluon plasma in heavy-ion reactions as shown in Fig. W. In addition, neutrino oscillation measurements become accurate and it is the stage to probe $\mathrm{CP}$ violation in the lepton sector. For this purpose, we need to understand neutrino-nucleus cross sections within about 5\% accuracy, for example, because the T2K target is water which contains the oxygen nucleus. The largest systematic error comes from neutrinonucleus interaction part, although the error is significantly reduced by near-detector measurements. T2K neutrino beam energies are not large enough to be sensitive to the DIS process; however, accurate nuclear PDFs (NPDFs) are important factors in Fermilab neutrino experiments in the several $\mathrm{GeV}$ region.

For example, nuclear modifications are measured for the carbon nucleus by the ratio $F_{2}^{C} / F_{2}^{D}$ in Fig. 田. In the small- $x$ region at $x<0.05$, there are negative modifications typically $5-10 \%$ for the carbon, and it is called shadowing. The virtual photon could fluctuate into a $q \bar{q}$ (or a vector meson state) at small $x$. The $q \bar{q}$ pair (or vector mesons) strongly interacts with surface nucleons, so that the projectile does not interact with internal nucleons, namely they are shadowed by the surface ones. The $q \bar{q}$ propagation length is estimated as $\lambda=1 /\left|E_{V}-E_{\gamma}\right|=2 v /\left(Q^{2}+M_{V}^{2}\right)=$

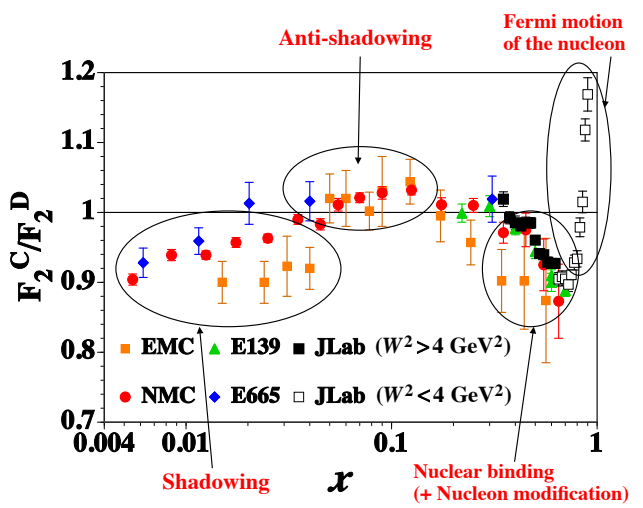

Figure 4: Nuclear modifications in $F_{2}^{C} / F_{2}^{D}$. $0.2 \mathrm{fm} / x>2 \mathrm{fm}$ at $x<0.1$. It becomes larger than the average separation of nucleons in a nucleus, and then multiple scattering occurs. Using multiple scattering theory, we can describe shadowing phenomena of $F_{2}$. At medium $x$, there are also negative nuclear modifications, and the ratio increases at large $x(>0.7)$ due to the nucleon Fermi motion. This part $(x>0.3)$ is described by the convolution picture. Namely, the nuclear structure function $F_{2}^{A}$ is calculated by the convolution of nucleon energy-momentum distribution in a nucleus with the nucleon structure function $F_{2}^{N}$. A slight shift in the energy-momentum distribution due to the binding energy results in the $5-10 \%$ modifications at medium $x$, and the Fermi motion modifies the function at large $x$. The modification is positive at $x=0.1$ and it is called anti-shadowing because of the opposite effect to the shadowing. It is necessary to have such positive effects to satisfy the baryon-number, charge, and momentum conservations for a nucleus, but its physics mechanism is not well studied so far.

There are two ways to determine the NPDFs. One is to obtain nuclear modification functions as typically shown in Fig. 团, and the other is to obtain the absolute NPDFs. The advantage of the first one is that we could possibly avoid obtaining unphysical NPDFs in the regions where there is no or few experimental data. Furthermore, the $x$ dependence of the nuclear modifications is roughly known and they are within 20-30\% even for large nuclei, and functional forms of the modifications could be roughly the same for all the partons. However, neutrino data are not provided in the ratio form e.g. with the deuteron, so that one has different treatments between the charged-lepton and neutrino data. The second method defines the absolute NPDFs on the same footing with the nucleon ones, which is the advantage as investigated by the nCTEQ collaboration. 
The PDF modifications are shown for the nCTEQ analysis in Fig. [ at $Q^{2}=2^{2} \mathrm{GeV}^{2}$ in comparison with other distributions [焑]. All the analysis results roughly agree with each other. The gluon modification is not well determined. Especially, the magnitude of the gluon shadowing is not very clear at this stage, although there could be a hint from $J / \psi$ production in the ultra-peripheral

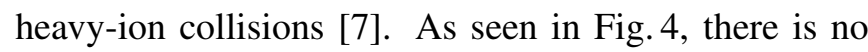
datum at $x<0.004$ for nuclear structure functions. However, the situation should be changed significantly by the EIC project where small- $x\left(\sim 10^{-3}\right)$ measurements will be done. Then, the scaling violation could constraint the gluon shadowing accurately. Furthermore, flavor dependent nuclear modifications could be measured at JLab by the parity-violating DIS and at Fermilab by Drell-Yan processes with nuclear targets.

\section{Polarized PDFs}

\subsection{Polarized PDFs of spin-1/2 nucleon}

The longitudinally-polarized PDFs have been investigated since 1980's, and we now know the quark and antiquark distributions from polarized DIS processes and polarized proton-proton collisions, although flavor dependent antiquark and gluon distributions still have large errors. It is known that the determined polarized PDFs could be changed depending on fragmentation functions if the semi-inclusive DIS data are included. In a recent JAM collaboration analysis, fragmentation function data are included into their analysis data set toward a "real" global analysis [ $[8]$. The determined polarized PDFs are shown in Fig. 6 with their uncertainties. The $\Delta u^{+}$and $\Delta d^{+}$distributions are well determined, but the antiquark ones have large errors at this stage.

The gluon polarization has not been determined accurately. There was a report in 2014 that a significant fraction $(\sim 40 \%)$ of nucleon spin could be carried by the gluon from an analysis with $\pi^{0}$-production measurements in the polarized pp collisions at RHIC. However, its uncertainty is still large as shown in Fig. $\square$ because the small- $x$ region has not been probed in any experiments so far. One of the major projects of EIC is to determine

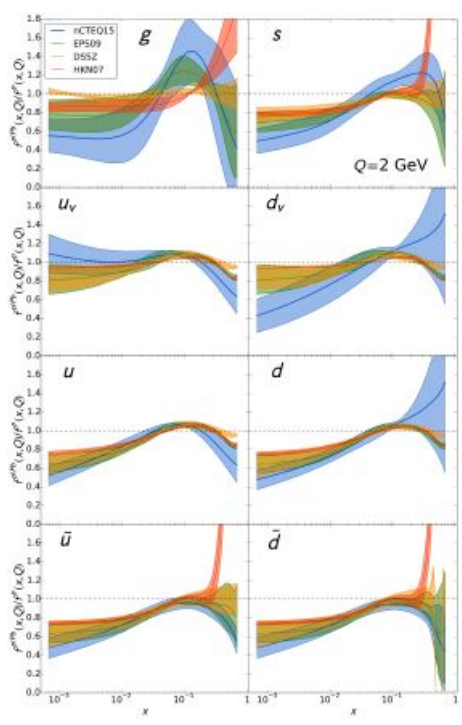

Figure 5: PDF modifications in iron [焑].
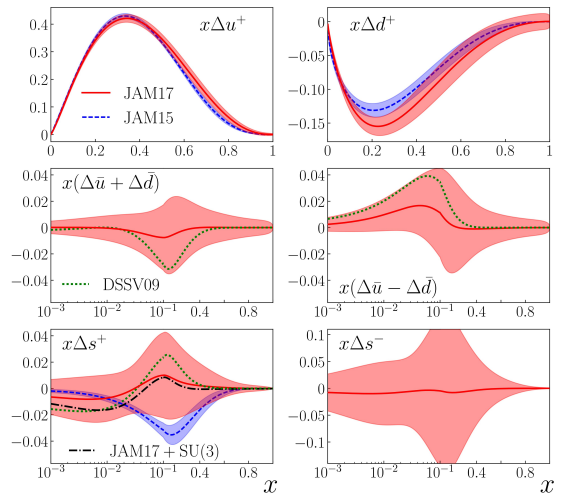

Figure 6: Polarized PDFs of nucleon [区]].

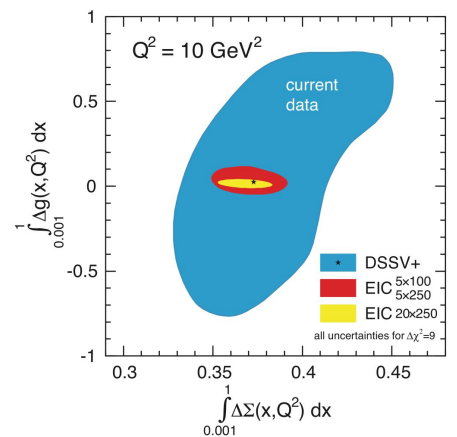

Figure 7: Gluon polarization at EIC [Q]]. 
the gluon-spin contribution to the nucleon spin precisely [Q]. In particular, scaling-violation measurements will impose a strong constraint for the gluon polarization, and it should be determined accurately by the future EIC data.

These are longitudinally-polarized PDFs, whereas there are transverse ones called transversity distributions. The quark transversity distributions have chiral-odd nature, and they are decoupled from the gluon one in the $Q^{2}$ evolution. Because transverse spin phenomena should be the same as the longitudinal one at low energies, their difference from the longitudinally-polarized PDFs should deepen our knowledge on nucleon spin. It is interesting that their $Q^{2}$ evolution difference from the longitudinally-

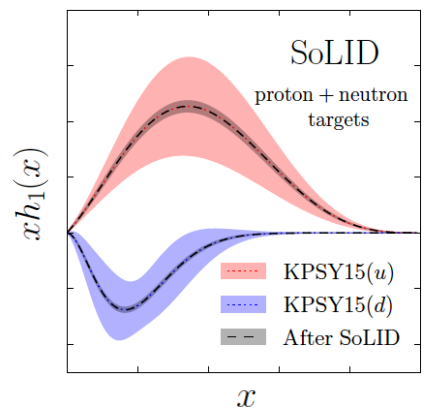

Figure 8: Transversity and SoLID [山]]. polarized PDFs probes a perturbative aspect of nucleon spin physics. The transversity distributions are determined precisely by the future SoLID (Solenoidal Large Intensity Device) experiment as shown in Fig. [1] [0]].

\subsection{Polarized PDFs of spin-1 deuteron}

It is known that the spin-1 deuteron has additional structure functions to the ones in the spin- $1 / 2$ nucleon. The twist- 2 ones are called $b_{1}$ and $b_{2}$, which are related with each other by the Callan-Gross type relation in the Bjorken scaling limit. The $b_{1}$ project will start soon at JLab [U]], whereas there are HERMES measurements as shown in Fig. Q.

The deuteron structure has been investigated for a long time at low energies and it is described as a bound system of proton and neutron in mainly $\mathrm{S}$ wave with small D-wave admixture. Using this standard model with a convolution formalism for the deuteron structure function $b_{1}$, we obtained the solid curve [ㅁ] , which is very different from the HERMES measurements, as shown in Fig. Q. Since the HERMES errors are large, we had better wait for the JLab measurement. However, the large difference could suggest that a new hadron-physics mechanism may be needed for explaining $b_{1}$ beyond the usual standard convolution model. A new interesting field of high-

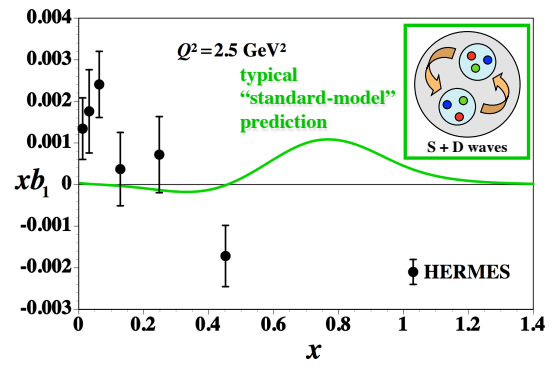

Figure 9: Standard convolution model for $b_{1}$ and HERMES data.

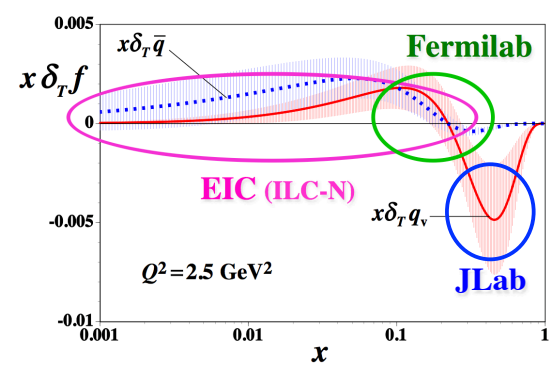

Figure 10: Future prospects on tensorpolarized PDFs. energy spin physics could be created by future $b_{1}$ measurements at JLab and EIC. In the parton model, $b_{1}$ is expressed by the tensor-polarized PDFs, which could be also measured at Fermilab by proton-deuteron Drell-Yan experiment E1039 [U]]. The kinematical regions of these future projects are shown together with possible tensor-polarized PDFs to explain the HERMES data [[13] in Fig. 四. 


\section{Fragmentation functions}

Fragmentation functions (FFs) indicate probabilities of parton fragmentation into hadrons, and they are used for calculating high-energy hadron reaction cross sections in Fig. W. They are determined mainly by hadron-production measurements in electron-positron annihilation. The Belle and $\mathrm{BaBar}$ collaborations published accurate data on the hadron productions at the center-of-mass energy of about $10 \mathrm{GeV}$. In comparison with previous measurements at SLD and LEP at the Z mass, the gluon fragmentation function is determined more accurately through the scaling violation [144].

In Fig. W, typical global analysis results [प5] are shown for the pion and kaon FFs. These functions are obtained by analyzing $e^{+} e^{-}$data, and they are compared with previous analysis results of HKNS and DSS. There are variations among the groups, but they are roughly consistent with each other within the uncertainties. We should note that a variation in strange fragmentation function could result in a significant change in the unpolarized and po-

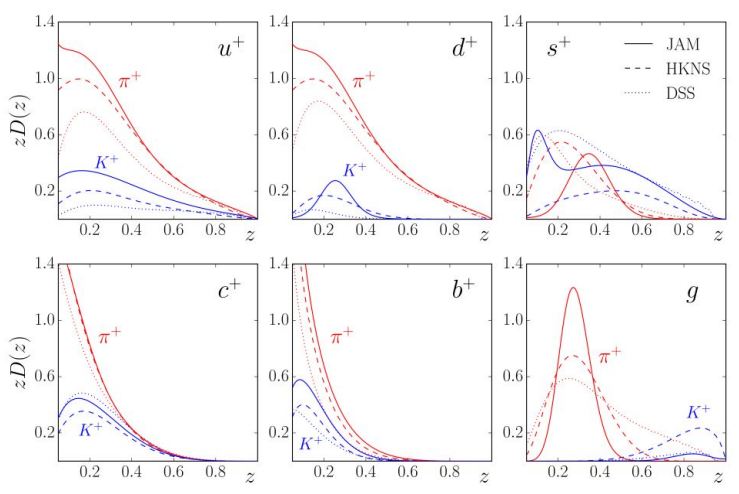

Figure 11: Determination of fragmentation functions [प[5]. larized strange-quark distributions in the nucleon. Therefore, for a precise determination of nucleonic PDFs in future, it is necessary to have accurate FFs because hadron-production data are included in many global analysis of PDFs.

\section{Hadron tomography by three-dimensional structure functions}

For clarifying internal structure of the nucleon, we have been investigating elastic form factors and the PDFs. In order to find the origin of the nucleon spin, orbital angular momenta of quarks and gluons become important factors and they could be probed by three-dimensional structure functions called generalized parton distributions (GPDs). The $s-t$ crossed quantities

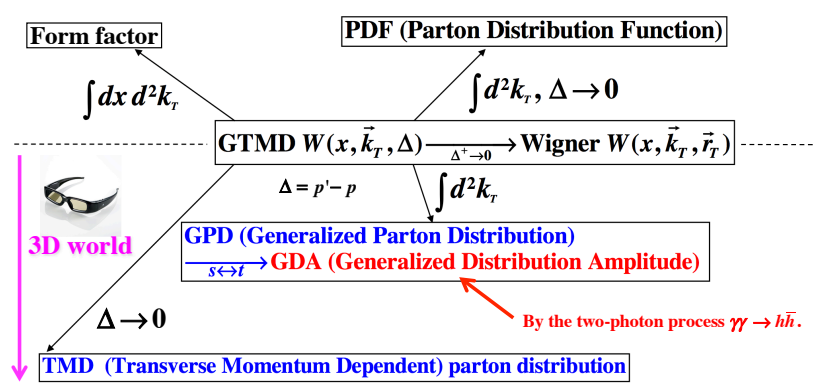

Figure 12: Wigner distribution and 3D structure functions. of the GPDs are generalized distribution amplitudes (GDAs). There are other popular 3D functions called transverse-momentum-dependent parton distributions (TMDs). The form factors, PDFs, GPDs, GDAs, and TMDs are obtained from the generating function, the Wigner distribution or generalized TMDs as shown in Fig. [2]. 


\subsection{Transverse-momentum-dependent parton distributions (TMDs)}

In Fig. [3], the TMD types are shown by indicating polarizations of nucleon and quark. We have already explained the unpolarized PDFs, the longitudinallypolarized PDFs, and the transversity distributions. There are corresponding TMDs $f_{1}, g_{1 L}$, and $h_{1 T}$. In addition, there are Boer-Mulders $\left(h_{1}^{\perp}\right)$, Sivers $\left(f_{1 T}^{\perp}\right)$, worm-gear $\left(g_{1 T}, h_{1 L}^{\perp}\right)$, pretzelosity $\left(h_{1 T}^{\perp}\right)$ distributions depending on the polarizations.

The unpolarized TMDs are obtained in Ref. [16], where the Gaussian function is assumed for the transverse distributions. Using semi-inclusive DIS, DrellYan, and Z-boson production data, they determined the TMDs by restricting the analysis to the low-transversemomentum region. A typical average transversemomentum squared $\left\langle k_{\perp}^{2}\right\rangle$ is shown as the function of $x$ in Fig. 14. At large $x$ where valence quarks dominate, the average $k_{T}^{2}$ is small, and it becomes larger at small $x$

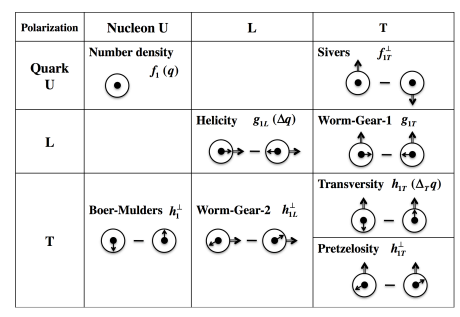

Figure 13: Various TMDs with nucleon and quark polarizations.

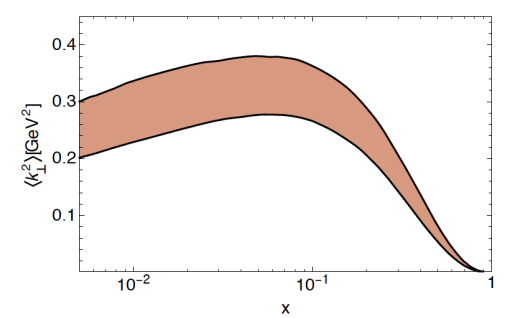

Figure 14: Transverse-momentum squared average [16] $]$. in the sea-quark region, and then it stays roughly the same at $x<0.1$.

Precision studies of the TMDs will be done by the JLab SoLID project. There are studies on determinations of the Sivers [ए]], Pretzelosity [ए8], and BoerMulders [ष्प]. However, the current distributions have large uncertainties in general, and the SoLID experiment will improve the situation drastically in the near future by accurate measurements. It is typically shown in Fig. [15, where the $u$-quark pretzelosity distribution is shown. Currently, the uncertainty is too large and we are

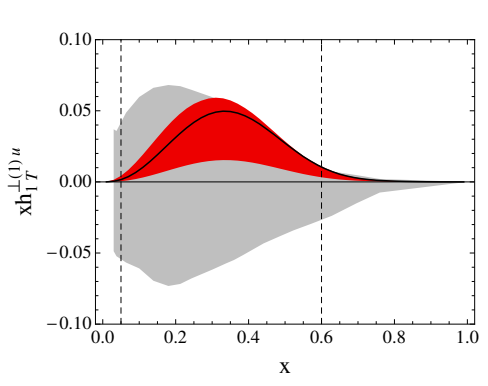

Figure 15: Pretzelosity and SoLId [20]]. not sure whether it is even positive or negative distribution. The SoLID project will certainly clarify the distribution with small uncertainties [ㅁ] . Furthermore, the EIC project probe the TMDs in a smaller- $x$ region. By these future experimental measurements, the TMD physics will be developed significantly in 2020's.

\subsection{Origin of nucleon spin by 3D structure functions}

We explained the situation of the origin of the nucleon spin, namely the longitudinally-polarized PDFs, in Sec.团. There is still large uncertainty in the gluon contribution. However, the remaining possibility is the effect of partonic orbital angular momenta. It can be determined experimentally by the GPDs which are measured in deeply virtual Compton scattering (DVCS). For example, the second moments of quark GPDs, $H_{q}(x, \xi, t=0)$ and $E_{q}(x, \xi, t=0)$, are related to the

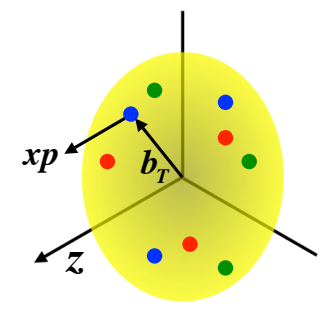

Figure 16: 3D view of hadron. 
orbital-angular-momentum contribution $L_{q}$ as

$$
\begin{aligned}
J_{q} & =\frac{1}{2} \int_{-1}^{1} d x x\left[H_{q}(x, \xi, t=0)+E_{q}(x, \xi, t=0)\right] \\
& =\frac{1}{2} \Delta q+L_{q},
\end{aligned}
$$

by finding the quark-spin contribution $\Delta q$ from other experiments. The Fourier transforms of the GPDs in the transverse coordinates in Fig. 16 indicate partonic transverse distributions as shown in Fig. [17.

Because the GPDs have three variables, it is difficult to determine accurately in the whole kinematical region. The DVCS cross section is given by the GPDs integrated over $x$, so that we need assumptions on their functional forms on $x, \xi$, and $t$, or by the GPDs with the constraint

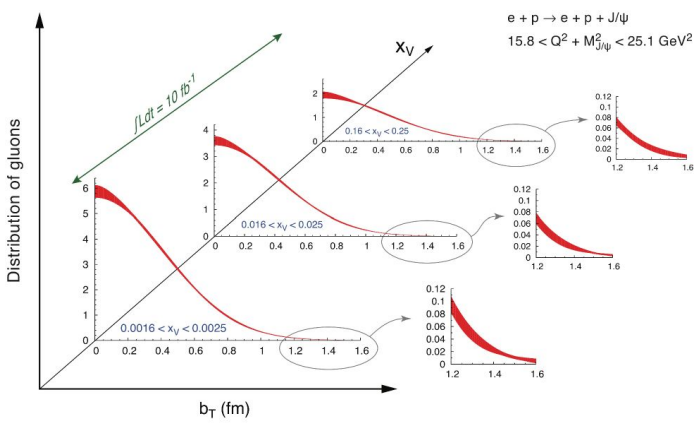

Figure 17: GPD studies at EIC [9]. $x=\xi$ or $-\xi$. The GPDs are measured by the virtual Compton scattering, and measurements are in progress in the JLab and COMPASS experiments. The current GPDs are not well constrained [R] $]$, so that it is one of major purposes of the future EIC project to determine them accurately. Furthermore, there are possibilities to measure the GPDs at hadron facilities such as J-PARC, for example, by using exclusive Drell-Yan $\pi^{-}+p \rightarrow \mu^{+} \mu^{-}+n$ and exclusive hadron reactions, e.g. $N+N \rightarrow N+\pi+B$ [22] at the high-momentum beamline under construction right now. In future, hadron facilities could provide an alternative way to probe 3D structure of hadrons.

The GPDs are determined by the DVCS $\gamma^{*} h \rightarrow \gamma h$ on the left-hand side of Fig. [18, and there are $s-t$ crossed quantities, the GDAs, as shown on the righthand side [23], 24]. The GDAs are measured in the two-photon process $\gamma^{*} \gamma \rightarrow$ $h \bar{h}$, which is possible at electron-positron collider such as KEKB and future ILC. The GPDs and GDAs are connected by

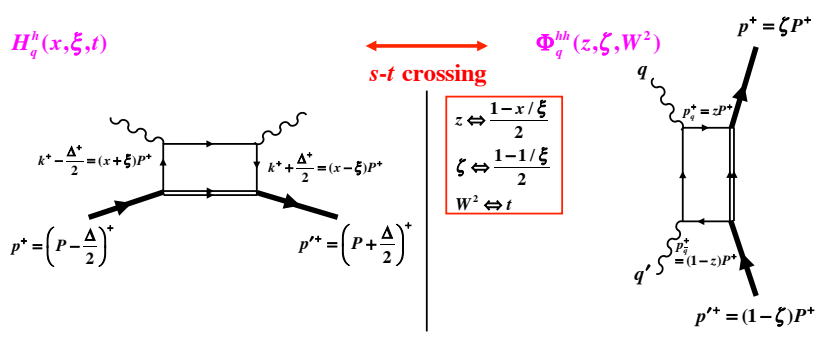

Figure 18: GPDs and GDAs. the $s-t$ crossing, so that both quantities are valuable for probing 3D structure of hadrons and origin of the nucleon spin.

\subsection{Tomography of exotic hadron candidates}

In the last decade, there have been reports on a number of exotic-hadron candidates. However, it is not obvious whether the findings indicate "real" exotic hadrons only by global observables such as masses, spins, decay widths, etc. Furthermore, because the quark number is not a conserved quantity, we need to consider a possible way to find exotic configurations. In this respect, highenergy hadron reactions could be useful tools to find the internal structure. For example, there is a method to find an internal constituent number involved in a hard exclusive reaction. It is called the constituent-counting rule in perturbative QCD [25]]. 
On the other hand, there are possibilities to use the 3D structure functions which we have been discussing in this section [23]]. A simple functional form of the GPDs could be written as $H_{q}^{h}(x, t)=q(x) F(t, x)$, where $F(t, x)$ is the transverse form factor at $x$. We can predict the valence-quark distributions of exotic hadrons in Fig. 19 by assuming the functional form $x f(x)=N x^{\alpha}(1-x)^{\beta}$ with the constraint of valencequark number, counting rule of perturbative $\mathrm{QCD}$, and momentum fraction carried by valence quarks. Because momenta carried by more quarks (4 or 5 ) in the

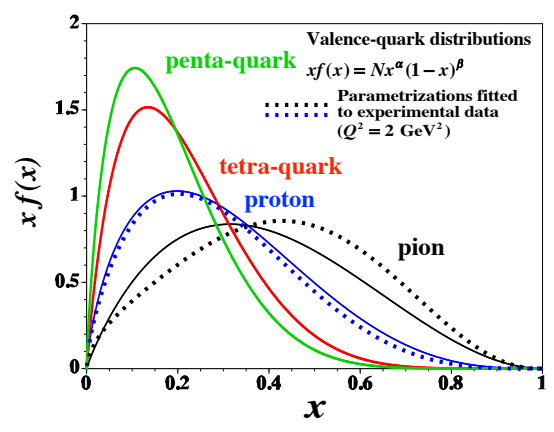

Figure 19: Valence-quark distributions of exotic hadrons [23] tetra- and penta-quark hadrons, the distributions shift to the smaller- $x$ region. The transverse spread should be also different depending on a compact quark-bound state or a diffusive molecular state, which could be observed in the transverse form factor of the GPDs. Of course, there is no stale fixed target for unstable exotic hadrons, so that such GPDs studies are not directly possible except for the transition GPDs. However, the exotic-hadron tomography should be possible by using the GDAs [[2]], which may be called timelike GPDs, because the exotic-hadron-pair productions are possible as shown in Fig. [8]. Because exotic-hadron cross sections are small in general, it is not easy but it could be an interesting future project.

\subsection{Origin of gravitational sources and hadron masses}

We discussed the studies on the origin of nucleon spin in polarized DIS and DVCS. The nucleon spin is defined by the matrix element of the angular-momentum tensor expressed by the energy-momentum tensor, whereas the nucleon mass is defined by the matrix element of the energy-momentum tensor as shown in Fig. [20. Therefore, studies to find the origin of nucleon spin are analogous to the ones of the origin of nucleon mass. For the last twenty

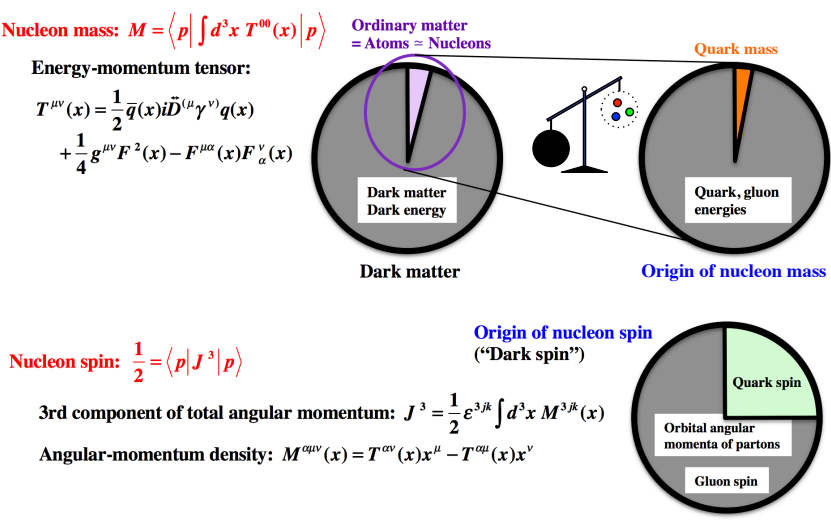

Figure 20: Origins of nucleon mass and spin. years, there have been discussions on the gauge-invariant decomposition of nucleon spin, and such studies settled down recently. The origin of nucleon mass has been also discussed, but they are mainly by effective hadron models. However, time has come to clarify it in terms of QCD with experimental confirmations.

As a part of such studies, we can investigate gravitational form factors which indicate mass, pressure, and shear-force distributions in hadrons in terms of quark and gluon degrees of freedom. Gravitational interactions are too weak to be studied directly in scattering experiments, so that they had been considered as a purely theoretical topic. However, there is a way to probe the gravitational form factors by the GPDs and GDAs. The GPDs and GDAs are defined by the same non-local 
vector operator, and their moments are given as

$\left.2\left(\frac{P^{+}}{2}\right)^{2} \int_{0}^{1} d z(2 z-1)^{n-1} \int \frac{d y^{-}}{2 \pi} e^{i(2 z-1) P^{+} y^{-} / 2} \bar{q}(-y / 2) \gamma^{+} q(y / 2)\right|_{y^{+}=\vec{y}_{\perp}=0}=\bar{q}(0) \gamma^{+}\left(i \overleftrightarrow{\partial^{+}}\right)^{n-1} q(0)$

For $n=2$, this operator is the energy-momentum tensor of a quark, and it is a source of gravity, whereas it is the vector-type electromagnetic current for $n=1$, as shown in Fig. [2] [24].

Then, using KEKB experimental data, for example, for the two-photon process $\gamma^{*} \gamma \rightarrow \pi^{0} \pi^{0}$, we determined timelike gravitational form factors, which are then converted to spacelike form factors by using a dispersion relation. They indicate mass (energy) distribution as well as the pressure and shear-force distributions in the pion as shown in Fig. 222, and they are obtained from two gravitational form factors $\Theta_{1}$ and $\Theta_{2}$. From these form factors, we calculated the mass (energy) radius and the mechanical (pressure and shear force) radius from $\Theta_{2}$ and $\Theta_{1}$, respectively. They are calculated as $\sqrt{\left\langle r^{2}\right\rangle_{\text {mass }}}=0.56 \sim 0.69 \mathrm{fm}$, whereas the mechanical radius obtained from $\Theta_{1}$ is larger $\sqrt{\left\langle r^{2}\right\rangle_{\text {mech }}}=1.45 \sim 1.56 \mathrm{fm}$ [24]. This is the

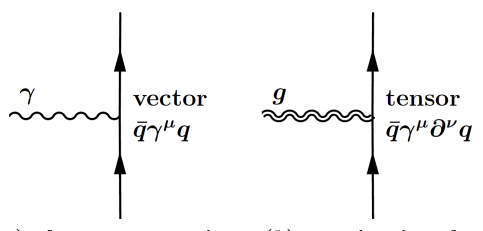

(a) electromagnetic (b) gravitational

Figure 21: Electromagnetic and gravitational interactions.

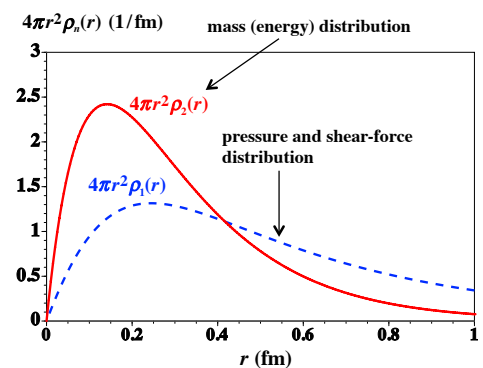

Figure 22: Mass and mechanical (pressure /shear-force) distributions in pion. first report on the gravitational radii of a hadron from actual experimental measurements. It is interesting to find the possibility that the gravitational mass and mechanical radii could be different from the experimental charge radius $\sqrt{\left\langle r^{2}\right\rangle_{\text {charge }}}=0.672 \pm 0.008 \mathrm{fm}$ for the charged pion. There are also recent studies on the gravitational form factors [26]. The KEKB was just upgraded, so that much accurate measurements will be obtained in the near future for the GDAs. We expect that the GDA studies will become popular as the GPD ones. Similar studies should be possible by the GPDs, especially if accurate DVCS measurements will be done at JLab and EIC.

\subsection{Nuclear composition of ultra-high-energy cosmic rays}

Ultra-high-energy cosmic ray physics is interesting not only for studying the cosmic microwave background, especially for confirming the GZK (Greisen-Zatsepin-Kuzmin) cutoff, but also for hadron physics on small- $x$ and forward physics. Another important connection, especially to the hadron tomography, is the determination of nuclear composition of ultra-high-energy cosmic rays. The nuclear composition, namely whether the cosmic ray is proton or heavier nuclei such as iron, is determined by the slant depth of shower maximum $X_{\max }$ in the simulation of air shower by taking into account cosmic-ray measurements on the earth surface. There are experimental indications that the ultra-high-energy cosmic rays are light nuclei according to recent Auger results in the energy region $10^{17}-10^{20} \mathrm{eV}$. Although typical simulation codes, EPOS, SIBYLL, QGSJET, and DPMJET indicate similar energy dependencies, the situation could change by the hadron-tomography studies. It is typically shown in Ref. [27] that the energy dependence could change depending on the transverse structure of the nucleon. Currently, studies on the 3D structure functions GPDs, 
GDAs, and TMDs are in progress as explained in this article. We expect that their relations with the cosmic-ray phenomena should be investigated further in the near future, as well as the studies of small/large- $x$ physics and Regge/Pomeron physics.

\subsection{Color entanglement}

As the TMD studies develop, color flow became important for the first time in experiments of hadron physics. We know that the color gauge invariance is satisfied by the intermediate gauge link $U$ as

$$
q\left(x, k_{\perp}\right)=\left.\int \frac{d z^{-} d^{2} z_{\perp}}{2(2 \pi)^{3}} e^{-i x p^{+} z^{-}+i \vec{k}_{\perp} \cdot \vec{z}_{\perp}}\left\langle p\left|\bar{\psi}\left(z^{-}, \vec{z}_{\perp}\right) \gamma^{+} U\left(z^{-}, \vec{z}_{\perp} ; 0\right) \psi(0)\right| p\right\rangle\right|_{z^{+}=0}
$$

in defining the TMDs. The color flow given by the gauge link does not play an important role in the 1D quantities such as the longitudinal PDFs. However, it become conspicuous in the TMDs because of additional flow in the transverse direction as illustrated in Fig. 233. The paths are different between the semi-inclusive DIS and Drell-Yan, which results in the sign difference in their TMDs. This is the first case where the color flow appears in actual observables, and its experimental confirmation is in progress. It suggests an interesting future development to create a new field with explicit color degrees of freedom.

In obtaining the PDFs, we use the experimental data which satisfy kinematical conditions for factorization. For example, the cross section of the hard hadron production $p+p \rightarrow h+X$ in Fig. is factorized into $\sigma=\sum_{a, b, c} f_{a}\left(x_{a}, Q^{2}\right) \otimes f_{b}\left(x_{b}, Q^{2}\right) \otimes \hat{\sigma}(a b \rightarrow c X) \otimes D_{c}^{h}\left(z, Q^{2}\right)$. The color entanglement means that particles interacts with each other by strong (color) interactions and the state of each particle cannot be described independently from other-particle states even though the particles are separated by a large distance. It indicates that the color entanglement should be investigated as one of factorization-breaking effects. Because quantum entanglement is getting popular in various fields of science and technology, the color entanglement is an interesting interdisciplinary topic. With the development of TMD physics, the color entanglement became an interesting subject although it is not easy to find such a signature. For example, a possible factorization breaking process is shown in Fig. [24 for the di-jet production $p+p \rightarrow j_{1}+j_{2}+X$ [28]. The processes $p+p \rightarrow \gamma+h^{ \pm}+X, \pi^{0}+h^{ \pm}+X$ were experimentally investigated to find the color entanglement; however, it is inconclusive at this stage for such a signature. We need much better understanding on TMD physics to push this project in future.

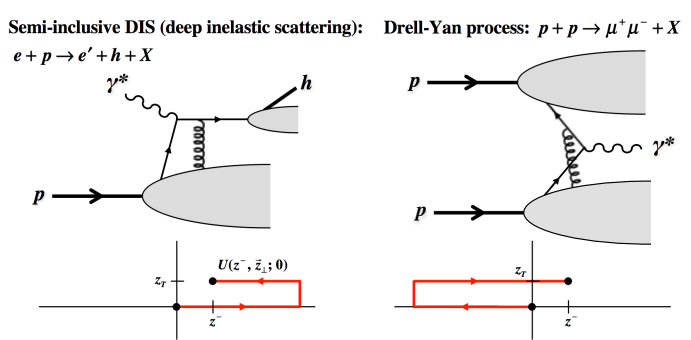

Figure 23: Gauge link and color flow.

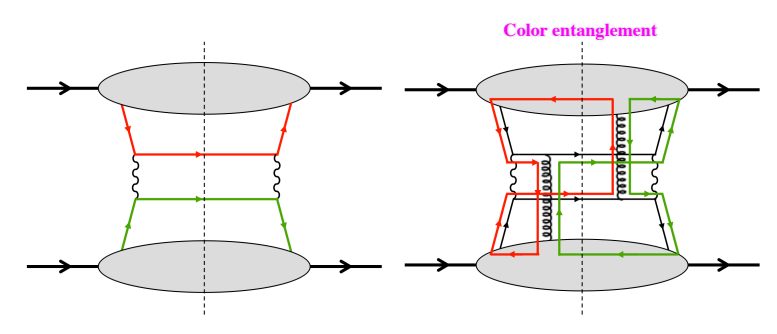

Figure 24: Color entanglement. 


\section{Summary}

The unpolarized PDF studies of the nucleon have a long history of about a half century. Now, they became a field of precision physics by a wide variety of high-energy experimental measurements together with detailed higher-order calculations in perturbative QCD. In addition, the field expanded to other topics, longitudinally- and transversely-polarized PDFs, tensor-polarized PDFs, nuclear PDFs, fragmentation functions, and 3D structure functions of GPDs, GDAs, and TMDs. Using precise PDFs, we expect that new physics beyond the standard model will be discovered and that new quark-gluon plasma phenomena will be found. Furthermore, we believe that the origin of the nucleon spin will be solved and the 3D tomography of the nucleon will be clarified. The 3D tomography studies are also valuable for understanding the composition of ultra-high-energy cosmic rays and GZK cutoff phenomena. We also bear in mind that the PDF field should be developed along with progress in other fields. The nucleon spin and 3D tomography field is closely connected with the gravitational physics because of the similar matrix elements of the angular-momentum and energy-momentum tensors. The energy-momentum tensor part provides information on the gravitational form factors. It will lead to a clarification of the origin of hadron masses or gravitational sources by the quark and gluon degrees of freedom. An effect of the color gauge link appears in the TMDs, which may be a beginning of new field on explicit color degrees of freedom. For example, the color entanglement is an interesting phenomena although its experimental confirmation is not easy at this stage. With future experimental facilities such as EIC and $\mathrm{LHeC}$, we expect to have further progress of the PDF field.

\section{Acknowledgments}

Figures 3, 7, 14 and 17 are used under the Creative Commons license with the copyright permission of authors. Figures 5, 6, and 11 are used with the copyright permission of American Physical Society and authors. Figure 8 is used with the copyright permission of Elsevier with an author. Figure 15 was supplied by Tianbo Liu and Haiyan Gao.

\section{References}

[1] R. D. Ball et al. (NNPDF Collaboration), Eur. Phys. J. C 77, 663 (2017).

[2] For example, see J. Ablinger et al., Nucl. Phys. B 927, 339 (2018) \& 932, 129 (2018); S. Moch et al., JHEP 1710, 041 (2017).

[3] G. P. Zeller et al., Phys. Rev. Lett. 88, 091802 (2002); S. Kumano, Phys. Rev. D 66, 111301 (2002).

[4] X. Ji, Phys. Rev. Lett. 110, 262002 (2013). For recent progress, see e.g. T. Ishikawa et al., Phys. Rev. D 96, 094019 (2017); Huey-Wen Lin et al., Prog. Part. Nucl. Phys. 100, 107 (2018); Yu-Sheng Liu et al., arXiv:1807.06566.

[5] M. G. Aartsen et al. (IceCube Collaboration), Nature 551, 596 (2017); Erratum 554, 554 (2018).

[6] K. Kovarik et al. (nCTEQ Collaboration), Phys. Rev. D 93, 085037 (2016).

[7] V. Guzey et al., Phys. Lett. B 726, 290 (2013).

[8] J. J. Ethier, N. Sato, and W. Melnitchouk, Phys. Rev. Lett. 119, 132001 (2017).

[9] A. Accardi et al., Eur. Phys. J. A 52, 268 (2016).

[10] Z. Ye et al. (SoLID Collaboration), Phys. Lett. B 767, 91 (2017).

[11] Proposal to Jefferson Lab PAC-38 (PR12-11-110), J.-P. Chen et al. (2011); Fermilab E1039 experiment, LoI P1039 (2013); S. Kumano and Qin-Tao Song, Phys. Rev. D 94 (2016) 054022. 
[12] W. Cosyn, Yu-Bing Dong, S. Kumano, and M. Sargsian, Phys. Rev. D 95, 074036 (2017).

[13] S. Kumano, Phys. Rev. D 82, 017501 (2010).

[14] M. Hirai, H. Kawamura, S. Kumano, and K. Saito, Prog. Theor. Exp. Phys. $113 B 04$ (2016).

[15] N. Sato et al., Phys. Rev. D 94, 114004 (2016).

[16] A. Bacchetta et al., JHEP 06, 081 (2017); I. Scimemi and A. Vladimirov, Eur. Phys. J. C 78, 89 (2018).

[17] M. Anselmino et al., Euro. Phys. J. A 39, 89 (2009).

[18] C. Lefty and A. Proukudin, Phys. Rev. D 91, 034010 (2015).

[19] Z. Lu and I. Schmidt, Phys. Rev. D 81, 034023 (2010); V. Barone, S. Melis, and A. Prokudin, Phys. Rev. D 82, 114025 (2010).

[20] H. Gao, presentation at this conference. The distribution is from Ref. [18]].

[21] M. Diehl and P. Kroll, Euro. Phys. J. C 73, 2397 (2013); D. Mueller, Few Body Syst. 55, 317 (2014); K. Kumericki, S. Liuti, and H. Moutarde, Eur. Phys. J. A 52, 157 (2016); H. Moutarde, P. Sznajder, and J. Wagner, arXiv:1807.07620.

[22] S. Kumano, M. Strikman, and K. Sudoh, Phys. Rev. D 80, 074003 (2009). T. Sawada et al., Phys. Rev. D 93, 114034 (2016)

[23] H. Kawamura and S. Kumano, Phys. Rev. D 89, 054007 (2014).

[24] S. Kumano, Qin-Tao Song, and O. V. Teryaev, Phys. Rev. D 97 (2018) 014020.

[25] H. Kawamura, S. Kumano, T. Sekihara, Phys. Rev. D 88, 034010 (2013); Wen-Chen Chang, S. Kumano, T. Sekihara, Phys. Rev. D 93, 034006 (2016).

[26] V. D. Burkert, L. Elouadrhiri and F. X. Girod, Nature 557 (2018) no.7705, 396; M. V. Polyakov and P. Schweitzer, arXiv:1801.05858; K. Tanaka, Phys. Rev. D 98, 034009 (2018).

[27] L. Portugal and T. Kodama Nucl. Phys. A 837, 1 (2010).

[28] T. C. Rogers and P. J. Mulders, Phys. Rev. D 81, 094006 (2010); A. Adare etal. (PHENIX Collaboration), Phys. Rev. D 95, 072002 (2017). 